\title{
Oxidation and Bromodehydroxymethylation of Benzylic Alcohols Using $\mathrm{NaBrO}_{3} / \mathrm{NaHSO}_{3}$ Reagent
}

\author{
Chang Keun Lee, Bon-Suk Koo, Yong Sook Lee, Hye Kyung Cho, and Kee-Jung Lee" \\ Department of Industrial Chemistry, Hanvang Thiversity. Seoul 133-791, Korea \\ Received JHI 29,2002
}

Key Words : Oxidation, Bromedehyd droxy methỵ lation. Benæy lic alcohol, Sodium bromate, Sodium hỵdrogensullite

The oxidation of benzylic alcohols to benzaldehydes is an important organic reaction and a number of methods have been dereloped for this purpose. ${ }^{1.2}$ Whilst numerous reagents have been developed to effect this process. bromate anion has been used for oxidizing alcohols to aldehydes or ketones. $^{3-r / s}$ esters ${ }^{3}$ and carbosylic acids." Bromate anion was also known to oxidize sulfides to sulfoxides. ${ }^{*}$ hydroquinones or polyaromatics to quinones, ${ }^{8.9}$ thiols to disulfides. ${ }^{6.113}$ iodobenzenes to iodoxybenzenes. ${ }^{11}$ In addition. there are some other examples such as cleavage of carbohydrate benzyl ethers and benzylidene acetals. ${ }^{12}$ preparation of bromoly drin derivatives. ${ }^{1.3}$ oxidative deprotection of tetraly dropy ranyl ethers. ethylene acetals. ${ }^{14}$ aromatic bromination. ${ }^{15}$ and bromination of alkylbenzenes. ${ }^{1 / 4}$ Meanw hile. Ishii and co-workers examined an appropriate method for generating $\mathrm{HOBr}$ equivalents from $\mathrm{NaBrO}_{3}$ combined with various reducing agents ( $\mathrm{NaHSO}_{3} . \mathrm{Na}_{2} \mathrm{SO}_{3}, \mathrm{Na}_{2} \mathrm{~S}_{2} \mathrm{O}_{3}$. $\mathrm{Na}_{2} \mathrm{HPO}_{3}$. FeSO . and $\mathrm{H}_{2} \mathrm{C}_{2} \mathrm{O}_{4}$ etc.) and found that $\mathrm{NaHSO}_{3}$ was the best reducing agent. ${ }^{1.3}$ They also showed that $\mathrm{NaBrO}_{3} / \mathrm{NaHSO}_{3}$ reagent was an efficient oxidizing agent of primary alcohols to dimeric esters. diols to hydroxyketones and/or diketones. ${ }^{1-}$ and ethers to esters ${ }^{1 ?}$ in aqueous medium. Ho emphasized that bromate ion without any mediate was not capable of oxidizing benzy lic alcohols. ${ }^{+}$

Recently; we described that Oxone ${ }^{\bar{k}}$ and bromide ions have been used for the oxidation of benzylic alcohols to benzaldehydes ${ }^{18}$ and the bromodecarbonylation of benzaldehydes with an electron-donating substituent at para position to bromoarenes..$^{19}$ In this paper. we report the oxidation of benzylic alcohols to benzaldehydes and the bromodehydroxymethylation of benzylic alcohols with an electron-donating substituent at para position to bromoarenes using a mixture of equimolar amounts of $\mathrm{NaBrO}_{3}$ and $\mathrm{NaHSO}_{3}$.

Optimization of the reaction conditions revealed that simple stirring a solution of benzylic alcohol ( 1 equivalent). $\mathrm{NaBrO}_{3}$ (2 equivalents) and $\mathrm{NaHSO}_{3}$ (2 equivalents) in a 1:1 mixture of $\mathrm{CH}_{3} \mathrm{CN} / \mathrm{H}_{2} \mathrm{O}$ effected the formation of benzaldehyde and benzoic acid in $77 \%$ and $21 \%$ isolated yields within $1.5 \mathrm{~h}$. respectively: When one equivalent of $\mathrm{NaBrO}_{3}$ and $\mathrm{NaHSO}_{3}$ were employed benzaldehyde $(4+\%)$ and undesirable benzoic acid $(11 \%)$ were obtained again.

*Comesponding author. e-mail: leekjíthanyang.ackr
Further studies showed that this oxidation method could be applied to a wide range of benzylic alcohols as shown in Table 1.

The presence of electron-withdrawing groups in the aromatic ring has lowered the oxidation rates. Thus. $p$ nitrobenzyl alcohol was oxidized to aldehyde in $48 \%$ yield and to acid in $32 \%$ yield over $5 \mathrm{~h}$. However, an electron-rich aromatic having unshared electron-pair at para position $p$ methoxybenzyl alcohol. not only was the ring brominated in the 2-position but the 4-hydroxymethyl group was elimunated and replaced by bromine. 4-bromoanisole $(67 \%)$ and 2.4-dibromoanisole (12\%) being products isolated ${ }^{\Upsilon i}$ Simularly $p$-acetamidobelzzyl alcohol afforded t-bromoacetanilide (53\%) and 2.4-dibromoacetanilide (4\%). On the other hand. $m$-methoxybenzyl alcohol gave exclusively ring brominated product 4-bromo-3-methosybenzyl alcohol $(76 \%)^{-1}$ and a small amount of 4-bromo-3-methoxybenzaldehyde (3\%)." No 3-methoxybenzaldehyde and bromodehydroxymethylation product were observed. The oxidation of I-phenylethanol afforded acetophenone in $9 \% \%$ yield. and 1-phenyl1.2-ethanediol was oxidized to 2-hydroxyacetophenone in $87 \%$ yield..$^{-}$

A plausible mechanism of the bromodehydroxymethylation is shown in Scheme 1. The oxidation of benzylic alcohols by the hypobromous acid affords benzaldehydes. which presumably proceed ipso-bromination and followed by nucleophilic attack of hydroxide ion or water on the aldehyde and subsequent elimination of formic acid to give bromoarenes. 19.23

In conclusion, we have shown in the present study that facile bromodehydroxymethylation of benzylic alcohols bearing para-electron donating substituents having unshared electron-pair can be carried out using a mixture of $\mathrm{NaBrO}_{3}$ and $\mathrm{NaHSO}_{3}$. However, other simple benzylic alcohols were oxidized to form benzaldehydes and benzoic acids.

\section{Experimental Section}

Melting points were determined in open capillaries with an Electrothermal melting point apparatus and are uncorrected. Progresses of reactions were followed by TLC using silica gel with fluorescent indicator coated on aluminium sheets. Infrared spectra were recorded on a Nicolet Magna 550 FTIR spectrometer and ' $\mathrm{H}$ NMR spectra were measured 
Table 1. Oxidation and Bromodehñ droxymethylation of Bencylic Alcohols Using $\mathrm{NaLrO}_{3}$ and $\mathrm{NaHSO}_{3}$

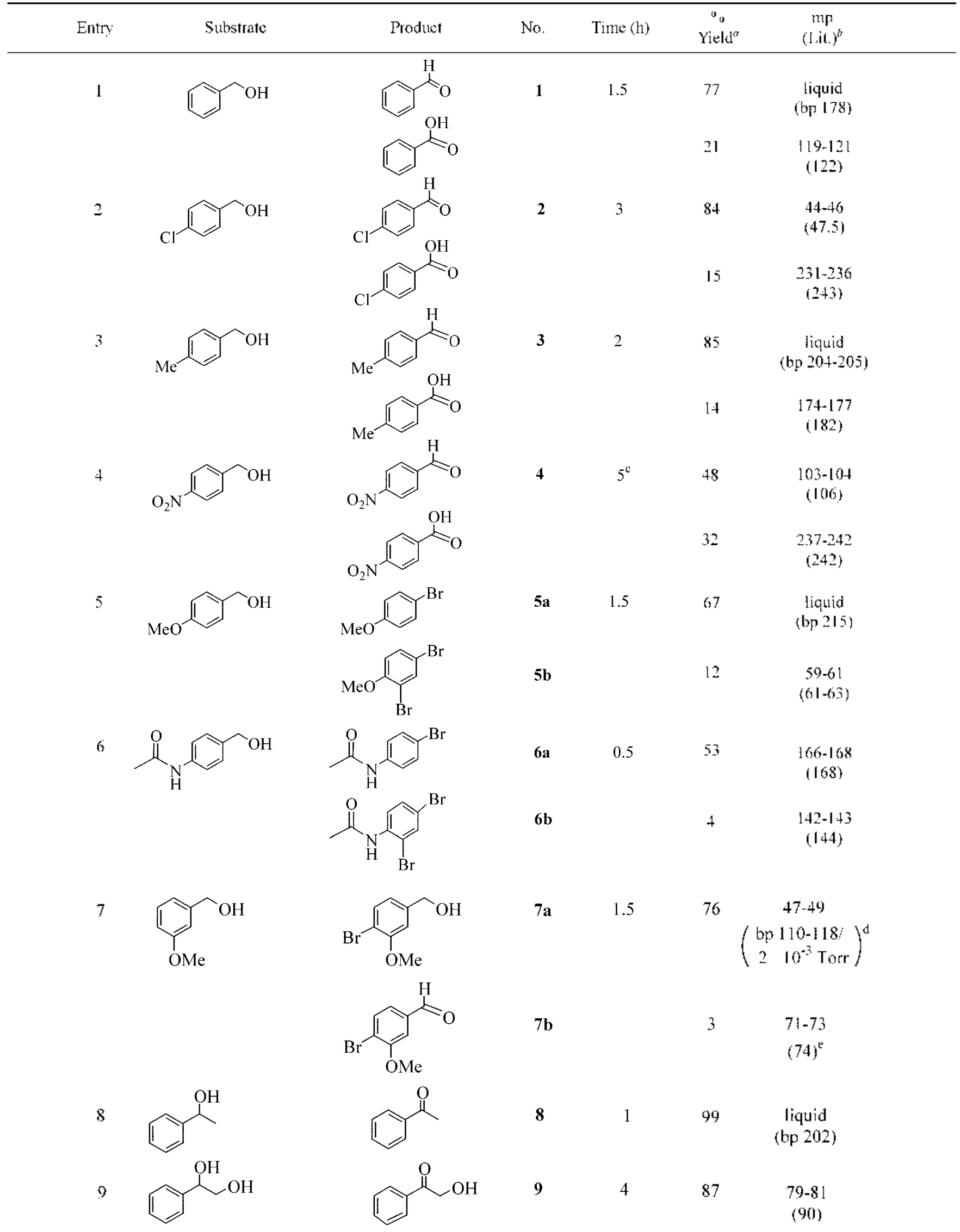




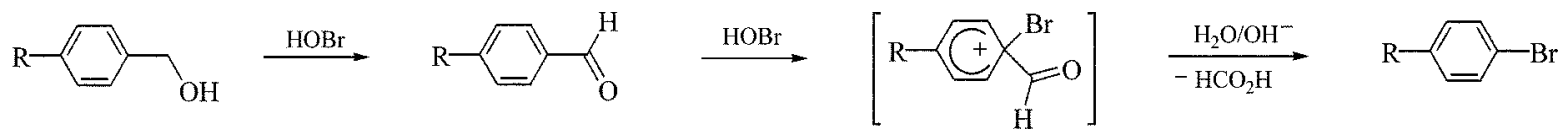

Scheme 1

on a Varian Gemini 300 spectrometer in $\mathrm{CDCl}_{3}$ using TMS as an intermal standard. Mass spectra were obtained on a ThemnoQuest Polaris Q mass spectrometer operating at 70 $\mathrm{eV}$.

General Procedure for the Reaction of Benzylic Alcohols with $\mathrm{NaBrO}_{3}$ and $\mathrm{NaHSO}_{3}$. To a stirred solutions of alcohols (5 munol) in aqueous $\mathrm{CH}_{3} \mathrm{CN}(30 \mathrm{~mL}, 1: 1 \mathrm{by}$ volume) was added $\mathrm{NaBrO}_{3}(1.5 \mathrm{lg} .10 \mathrm{mmol})$ and $\mathrm{NaHSO}_{3}$ ( 1.04 g. 10 mmol). Reactions were continuously monitored by thin-layer cluromatography and stirred at r.t. for the time indicated in Table 1 . The reaction mixture was quenched with aqueous sodium thiosulfate. and extracted with ether (3 $\times 30 \mathrm{~mL}$ ). The combined organic layers were washed with aqueous $\mathrm{Na}_{2} \mathrm{CO}_{3}$, water, dried over anhydrous $\mathrm{MgSO}_{1}$. filtered. and concentrated in vactio. The residue was chromatographed on a silica gel column and eluted with hexane-EtOAc 10:1 to give aldelydes or aryl bromides (Table 1). The combined aqueous layer was acidified with a $10 \% \mathrm{HCl}$ solution to $\mathrm{pH} 2$ and extracted with EtOAc $(2 \times 50$ $\mathrm{mL}$ ). The organic layers were washed with water. dried and exaporated to afford acid products.

The spectral data of products are as follows:

1: IR (neat) $\mathrm{cm}^{-1}: 1701.1600,1460.1312 .1204,827,749$ : ${ }^{3} \mathrm{H}$ NMR $\delta 7.45-7.67(\mathrm{~m} .3 \mathrm{H}) .7 .87-7.90(\mathrm{ml}, 2 \mathrm{H}), 10.02(\mathrm{~s}$. 1H): MS m/z (rel intensity) 106 (M'.34), 105 (74). 77 (100). $51(22)$.

2: IR (KBr) cm$~^{-1}: 1697,1576,1479,1386,1204.1013$. $811.539 .477:{ }^{1} \mathrm{H}$ NMR $\delta 7.53(\mathrm{~d}, J=8.5 \mathrm{~Hz}, 2 \mathrm{H}) .7 .83(\mathrm{~d} . J$ $=8.5 \mathrm{~Hz}, 2 \mathrm{H}), 9.99$ (s. $1 \mathrm{H}): \mathrm{MS} \mathrm{m} / \mathrm{z}$ (rel intensity) $\mathrm{l}+2\left(\mathrm{M}^{\prime}\right.$. 17). $141(58) .140\left(\mathrm{M}^{\prime} .46\right) .139(100) .113(7) .111(19) .77$ (6). $75(13)$.

3: $\mathrm{IR}$ (neat) $\mathrm{cm}^{-1}: 1701,1607.1386 .1308 .1207 .1164$, 847. 808: ${ }^{1} \mathrm{H}$ NMR $\delta 2.43$ (s. $3 \mathrm{H}$ ). 7.33 (d. $J=7.9 \mathrm{~Hz} .2 \mathrm{H}$ ). 7.78 (d. $J=7.9 \mathrm{~Hz} .2 \mathrm{H}$ ). 9.97 (s. $1 \mathrm{H}$ ); MS m/z (rel intensity) $120(\mathrm{M}, 40) .119(100), 91(72) .65(28)$.

4: IR $(\mathrm{KBr}) \mathrm{cm}^{-1}: 1712,1607,1538,1344,1293.1196$. $854.819 .738:{ }^{\prime} \mathrm{H}$ NMR $\delta 8.08(\mathrm{~d}, J=8.5 \mathrm{~Hz}, 2 \mathrm{H}$ ). $8.4 \mathrm{l}(\mathrm{d} . J$ $=8.5 \mathrm{~Hz} .2 \mathrm{H}$ ), 10.17 (s, $1 \mathrm{H})$. MS $\mathrm{m} / \mathrm{z}$ (rel intensity) $15 \mathrm{I}$ $\left(\mathrm{M}^{\prime}, 44\right), 150(100) .77(13), 51(16)$.

5a: IR (neat) $\mathrm{cm}^{-1}: 1577.1487 .1289,1239,1172.1033$. 823: ${ }^{1} \mathrm{H}$ NMR $\delta 3.77$ (s, 3H), 6.78 (d. $\left.J=8.9 \mathrm{~Hz} .2 \mathrm{H}\right) .7 .37$ (d. $J=8.9 \mathrm{~Hz} .2 \mathrm{H}$ ): MS $\mathrm{m} / \mathrm{z}$ (rel intensity) 188 (M'.98). 186 $\left(\mathrm{M}^{\prime}, 100\right), 173(31), 171(30), 145(23) .143(26) .77(31), 63$ (47).

5b: IR $(\mathrm{KBr}) \mathrm{cm}^{-1}: 1576,1475,1378,1263,1052.807$. 679. 617: 'H NMR $\delta 3.87$ (s. $3 \mathrm{H}), 6.77$ (d. $J=8.8 \mathrm{~Hz} . \mathrm{lH}$ ). 7.37 (dd, $J=8.8 .2 .3 \mathrm{~Hz} .1 \mathrm{H}) .7 .66($ d. $J=2.3 \mathrm{~Hz} . \mathrm{IH})$; MS $\mathrm{m} / \mathrm{z}$ (rel intensity) $268\left(\mathrm{M}^{\prime}, 35\right) .266\left(\mathrm{M}^{\prime} .75\right) .264\left(\mathrm{M}^{\prime} .42\right)$. 253 (9). 251 (18). 249 (20). 225 (16). 223 (35). 221 (16). 172 (15). $170(14), 63(100)$.

6a: $\operatorname{IR}(\mathrm{KBr}) \mathrm{cm}^{-1}: 3293,1677,1603,1526.1483,1394$.
$1305,1254,1013,823,737.504:{ }^{1} \mathrm{H}$ NMR $\delta 2.04$ (s, 3H). $7.47(\mathrm{~d} . J=8.9 \mathrm{~Hz}, 2 \mathrm{H}) .7 .56(\mathrm{~d} . J=8.9 \mathrm{~Hz}, 2 \mathrm{H}) .10 .07$ (s. lH); MS m/z (rel intensity) $215(\mathrm{M} .43), 213(\mathrm{M}, 43) .173$ (96). $171(100), 92(96), 65(41)$

6h: $\left[R(\mathrm{KBr}) \mathrm{cm}^{-1}: 3289.1658,1572.1522 .1460,1367\right.$. $1293,1040,831,602,547:{ }^{1} \mathrm{H}$ NMR $\delta 2.24(\mathrm{~s}, 3 \mathrm{H}), 7.42$ (dd. $J=8.9 .2 .1 \mathrm{~Hz}, \mathrm{lH}) .7 .57(\mathrm{~s}, \mathrm{lH}) .7 .68(\mathrm{~d} . J=2.1 \mathrm{~Hz}, \mathrm{lH})$. $8.26(\mathrm{~d} . J=8.9 \mathrm{~Hz}, \mathrm{lH}): \mathrm{MS} \mathrm{m} / \mathrm{z}$ (rel intensity) $295\left(\mathrm{M}^{\prime}\right.$. 12). 293 (M'. 20). 291 (M . 10). 253 (47). 251 (100). 249 (54). $214(70), 212(75) .172(31) .170(37), 91(36), 90(69)$, $63(44)$.

7a: IR (KBr) $\mathrm{cm}^{-1}: 3417,1596,1572,1471,1293.1266$, 1234, 1188. 1161, 1130. 1052, 1009. 854, 803: ' H NMR $\delta$ 2.62 (s. 1H). 3.78 (s. 3H), 4.66 (s. $2 \mathrm{H}) .6 .69$ (dd. $J=8.6,3.1$ Hz. lH). 7.03 (d. $J=3.1 \mathrm{~Hz}, \mathrm{lH}) .7 .38(\mathrm{~d} . J=8.6 \mathrm{~Hz}, \mathrm{lH})$; MS $\mathrm{m} / \mathrm{z}$ (rel intensity) $218(\mathrm{M} .89), 216\left(\mathrm{M}^{\prime}, 100\right), 137$ (57). $109(72) .94(20)$

7h: IR $(\mathrm{KBr}) \mathrm{cm}^{-1}: 1673.1592,1467,1285,928,819$. 648. 601: 'H NMR $\delta 3.85$ (s. $3 \mathrm{H}) .7 .05$ (m, lH). $7.4 \mathrm{l}(\mathrm{m}$, lH). 7.52 (m. IH). 10.32 (s. IH): MS m/z (rel intensity) 216 (M , 100), 2l+(M, 99). $215(95) .213(89)$.

8: IR (neat) $\mathrm{cm}^{-1}: 1681,1596.1448,1359.1262,951,765$, 687: ${ }^{1} \mathrm{H}$ NMR $\delta 2.60$ (s, 3H), 7.43-7.59 (m. 3H). 7.94-7.98 (m. 2H); MS m/z (rel intensity) 120 (M , 16). 105 (100), 77 (23). 51 (9).

9: $\mathrm{IR}(\mathrm{KBr}) \mathrm{cm}^{-1}: 3+21.1689,1600,1+56,1409,1301$, 1231. 1106.970.761.683: 'H NMR $\delta 3.51(\mathrm{t} . J=4.6 \mathrm{~Hz}$. IH). 4.89 (d. $J=4.6 \mathrm{~Hz} .2 \mathrm{H}) .7 .49-7.67$ (m. $3 \mathrm{H}) .7 .92-7.95$ (m. $2 \mathrm{H}$ ); MS m/z (rel intensity) 136 (M'. 1). 105 (77). 77 (100), $51(17)$.

\section{References}

1. (a) Iollingwortl, G. J. In Comprohonsive Organic Finctional (Wout Transfomations: Katritzky. A. R. Meth-Coln. O.. Rees. C. W.. Paltenden. G.. Eds.: Elsevier Science Lid.: Oxford. 1995: Vol. 3. pp 81-109. (b) Larock. R. C. Comprehensive Organic Thansformations: VCH Publishers. Inc.: New York, 1989; pp 604614

2. (a) Brink, G.-I. T.: Arends, I. W. C. F.: Sheldon, R. A. Science 2000. 287. 1636. (b) Barrell. A. G. M. Braddock. D. C.: McKinnell. R. M.: Waller. F. I. Synlet 1999. 1489. (c) Sato. K.: Takagi. J.: Aoki. M.: Noyori. R. Tetholedmm Tett. 1998. 39. 7549. (d) 7ondervan, C.: IJage, R.: Feringa. B. I. Chem. Commm. 1997, 419. (c) Barlate, N. B.: Sasidharan. M.: Sudalai. A.: Wakliarkar, R. D. Tetrohedron Lett 1996. 37. 2067. (1) Feldbcrg. L.: Sasson. Y. (Them. Commm. 1994. 1807.

3. (a) Tomioka. H.: Oshima. K.: Nozaki. H. Tetwahedron Tent. 1982. 23. 539. (b) Yamamoto. Y.: Suzuki. H.: Moro-oka. Y. Te'tratedm Lett. 1985, 26, 2107. (c) Kanemoto. S.: Tomioka, Il.: Oshima. K. Norati, It. Bull. Chem. Soc. Jph. 1986, 59,105

4. IHo. T.-I. Swhlhesis 1978, 9.36

5. (a) Farkas. L.: Schächter. O.J.1m. (7h'm. Soc. 1949.7/. 2827. (b) Kajigaeshi. S.: Nakagawa. T.: Nagasaki. N.: Yamasaki. H: 
Fujisuki. S. Bull. Chem. Soc. Jpn. 1986. 59.747.

6. Firoucabadi. H.: Mohammadpoor-Ballork. 1. Buhl. Chem. Soc. Jph. 1995. 6is. 2319.

7. Takase. K.: Masuda. II.: Kai. O.: Nishivama. Y: Sakaquchi. S.; Ishii, Y. Chem. Lett. 1995, 871. and only one example of conversion of benzyl alcohol to benzaldehyde $\left(5 l^{\circ} 0\right)$ and benzoic acid $\left(17^{\circ} 0\right)$ was reported.

8. Ho. I.-L. Symth. Commum. 1979.9.237.

9. Banerjee. A.: Dutl. S.: Sengupla. D.: Adak. M. M.: Samaddar. H. J. Ind Chem. Soc: $1983,60.275$

10. Adak. M. M: Banerjec. (i. C: Banerjec. A. J. Int Ch'm. Soc: $1985,62,224$.

11. Banerjee. A.: Banerjee. G. C.: Bhallacharya. S.: Banerjee. S.: Samaddar. H. J. Ind Chem. Soc. 1981. 5s. 605.

12. Adinolli. M.: Barone. Gi.: Guariniello. L.: Ladonisi. A. Tetrahedon Lett. $1999.40,84.39$.

13. Masuda IJ:- Takase. K: Nishio. M:- I Jasegawa. A: Nishiyama. Y.: Ishii. Y. J. O,g. Chm. 1994, 59.5550

14. Molammadpoor-Ballork. 1.: Nourozi. A. R. Symthesis 1999. 487.

15. Groweiss. A. Org. Process Re's. Deve 2000. t. 30.
16. Kikuchi. D.: Sakanuchi. S.: Ishii. Y.J. Org. (hem 1998. 63.6023,

17. Sakaguchi. S.: Kikuchi. D.: Ishii. Y. Buhl (hem. Soc. Jph 1997. 70. 2561

18. (a) Koo, B.-S.; L.e. C. K.: Lee. K.-J. Sinth. Commm, 2002, 32 , 2115 . (b) Bolm. C.: Magnus. A. S.: IIilderbrand. J. P. Org. Lett. 2000, 2. 1173 .

19. Koo. B.-S.: Kim. E.-H.: Lee. K.-I. Synth Commm 2002. 32. 2275 .

20. In the case of using one equivalent of $\mathrm{NaBrO}_{3}$ and $\mathrm{NaHSO}_{3}$. brommanisole $\left(48^{\circ} 0\right)$, 4-methoxybenzaldehyde $\left(2^{\circ} v\right)$ and unchanged t-methoxybenzel alcohol $\left(20^{\circ}\right.$ a) nere obtained.

21. Fllint, M.: Janes. N. F.: Pearson, B. C. J. Sci. Food Agric: 1967. 18. 325 .

22. (a) Hodgson. H. H.: Beard. H. G. J. Chem. Soc. 1925. 875. (b) Inokuchi. T: Matsumolo. S.: lorii. S. J. Org. (hem. 1991. 56. 2416.

23. For a similar chlorodehydroxymethylation reaction of $o$ hydroxybenzyl alcohol using hypochlorite solution. see: Meyers. C. Y.J. OH. ( $\%$ em 1961.26.1046. 